\title{
BMJ Open Burden, risk factors and outcomes of hyperemesis gravidarum in low-income and middle-income countries (LMICs): systematic review and meta- analysis protocol
}

Mesfin Tadese Dinberu, ${ }^{1}$ Mohammed Akibu Mohammed, ${ }^{1}{ }^{1}$ Tesfalidet Tekelab, ${ }^{2}$ Nigus Bililign Yimer, ${ }^{3}$ Melaku Desta, ${ }^{4}$ Tesfa Dejenie Habtewold ${ }^{\odot, 6}$

To cite: Dinberu MT, Mohammed MA, Tekelab T, et al. Burden, risk factors and outcomes of hyperemesis gravidarum in low-income and middleincome countries (LMICs): systematic review and metaanalysis protocol. BMJ Open 2019;9:e025841. doi:10.1136/ bmjopen-2018-025841

- Prepublication history and additional material for this paper are available online. To view these files, please visit the journal online (http://dx. doi org/10.1136/bmjopen-2018025841).

Received 13 August 2018 Revised 14 February 2019 Accepted 19 February 2019

D) Check for updates

(C) Author(s) (or their employer(s)) 2019. Re-use permitted under CC BY-NC. No commercial re-use. See rights and permissions. Published by BMJ.

For numbered affiliations see end of article.

Correspondence to Mesfin Tadese Dinberu; mesitad031@gmail.com

\section{ABSTRACT}

Introduction Hyperemesis gravidarum $(\mathrm{HG})$ is a pregnancy condition characterised by excessive nausea and vomiting resulting in dehydration, weight loss and serious adverse pregnancy outcomes including termination of pregnancies. Even though evidence in low-income and middle-income countries (LMICs) is limited, the prevalence of $\mathrm{HG}$ in pregnancy ranges from $0.3 \%$ to $10.8 \%$. With this systematic review and meta-analysis, we aim to determine the prevalence/burden, risk factors, and maternal and perinatal outcomes of $\mathrm{HG}$ in LMICs.

Methods PubMed, CINAHL, EMBASE, EBSCO, Ovid maternity and infant care databases, Cochrane Database of Systematic Reviews, Web of Science and SCOPUS databases will be searched. Reference lists of selected articles will be assessed in order to identify other potential studies of interest. Observational studies and (non) randomised controlled trials conducted from January 2000 to September 2018 in LMIC will be included. A weighted inverse-variance meta-analysis using fixed-effects and random-effects model will be done to generate a pooled estimate. Funnel plot and Egger's regression statistical test will be applied to check publication bias. Heterogeneity among studies will be checked using $\mathrm{T}^{2}$ to determine dispersion. Moreover, meta-regression analysis will be performed to investigate the source of heterogeneity. STATA V.14 will be used to analyse the data.

Ethics and dissemination Formal ethical approval and patient consent are not required; as primary data collection will not be employed. The result will be published in a peer-reviewed scientific journal and will be presented at scientific conferences and public press. PROSPERO registration number CRD42018096284.

\section{INTRODUCTION}

Nausea and vomiting of pregnancy (NVP), commonly known as morning sickness, is a minor disorder of pregnancy which usually disappears during the first trimester with occasional persistence until delivery. ${ }^{1}$ About $70 \%-80 \%$ of pregnant women experience some type of NVP. ${ }^{2}$ However, hyperemesis
Strengths and limitations of this study

- An extensive scoping review of hyperemesis gravidarum will be addressed.

- Quality assessment and statistical analysis scheme are powerful and robust.

- The review will address the latest published data.

- The lack of uniform diagnostic criteria may allow substantial heterogeneity and weakens comparability across studies.

- Missing of some relevant findings may occur due to language restriction.

gravidarum (HG) is a pregnancy condition characterised by prolonged and excessive/ severe NVP resulting in dehydration and weight loss that requires extensive medical care and hospital admission. ${ }^{3-5}$ The risk of admission for hyperemesis is found to be 29 times higher if the previous pregnancy was complicated by antenatal admission for hyperemesis. ${ }^{6}$ Estimates of HG vary across countries in which $0.3 \%-1.5 \%$ in high-income countries ${ }^{7}$ and $4.5 \%-10.8 \%$ in low-income countries. ${ }^{8}{ }^{9}$ This might be due to a lack of uniform diagnostic criteria, the higher percentages might be as a result of diagnosing a milder form of NVP. ${ }^{10}$ The risk of recurrence in subsequent pregnancies is reported to be $15 \% .^{11}$

Hyperemesis patients commonly have multiple pregnancies and current or previous molar pregnancy. ${ }^{12}$ Other risk factors for hyperemesis include maternal age, genetic susceptibility, parity, ethnicity, marital status, smoking, unplanned pregnancies, depression or psychiatric illness, less socioeconomic status, previous history of hyperemesis, pre-existing diabetes, body mass index, asthma, hyperthyroid disorders, female fetus, 
dysmenorrhea, urinary tract infections, peptic ulceration and other gastrointestinal disorders. ${ }^{6913-18}$

HG causes a wide range of maternal and fetal poor health outcomes that necessitate hospital admission if not treated properly. The more the severity of the symptoms, the greater the adverse outcomes. ${ }^{19}{ }^{20}$ Severe nutritional deficiencies including thiamine and vitamin $\mathrm{B}_{1}$, Mallory-Weiss syndrome, Wernicke's encephalopathy, hypocalcaemia and thyroid dysfunction are some of the major maternal sequels. ${ }^{21}{ }^{22}$ An increased risk of comorbidity, especially with feelings of depression, anxiety and heartburn, and reflux problems also pose a significant burden on women. ${ }^{23}{ }^{24}$ Similarly, the fetus is also at increased risk of future psychiatric disorders mainly anxiety, depression and bipolar disorder. ${ }^{25}$

Hyperemesis imposes a negative impact on health-related quality of life and daily life functioning. It also affects physical, social and emotional functioning, bodily pain, general health perception, vitality and mental health. A review of 38 studies concluded that hyperemesis leads to poor quality of life and negatively affects the social, occupational and domestic life functioning. ${ }^{26}$ In addition, a recent study in Norway shows that about $25 \%$ of women with HG consider terminating the pregnancy and $75 \%$ of them prefer not to get pregnant again. ${ }^{27}$ Furthermore, hyperemesis bears a substantial economic burden on women and their families. ${ }^{2} 2829$

Despite the high burden and risk of poor maternal and newborn health outcome, up-to-date evidence is lacking in low-income and middle-income countries (LMICs). Therefore, the purpose of this systematic review and meta-analysis is ${ }^{1}$ to estimate the prevalence of $\mathrm{HG}^{2},{ }^{2}$ identify risk factors of $\mathrm{HG}$ and ${ }^{3}$ investigate maternal and fetal health outcomes of HG in LMICs.

\section{METHODS}

\section{Protocol preparation and results reporting}

The protocol is written in line with the recommendations of Preferred Reporting Items for Systematic Reviews and Meta-Analyses (PRISMA) ${ }^{30}$ guideline (see online supplementary file 1) ${ }^{31}{ }^{32}$ Likewise, the results will be reported based on the PRISMA 2009 statement. $^{30}$ The article screening and selection process will also be demonstrated through a PRISMA flow diagram.

\section{PECO search guide}

$\checkmark$ P- Pregnant women in LMIC who diagnosed with HG regardless of the duration of pregnancy. LMICs will be identified according to the world bank new country classification by income level. ${ }^{33}$

$\checkmark \mathbf{E}$ - Exposure of HG includes any risk factors that are characteristics of the women including conditions before pregnancy, during pregnancy and fetal-related risk factors.

$\checkmark$ G- The comparison will be made across both within women with hyperemesis and between hyperemesis and non-hyperemesis women. For example, the effect of parity within women with hyperemesis, and between hyperemesis and non-hyperemesis women (general obstetric population).

$\checkmark \mathbf{O}-\mathrm{HG}$ and it is maternal and fetal poor health outcomes such as depression, bipolar disorder, anxiety and heartburn, Mallory-Weiss syndrome and Wernicke's encephalopathy. HG is the extreme/severe form of NVP. To diagnosis HG, two certain clinical criteria should be met. These include pregnancy related, persistent NVP not caused by other underlying medical conditions, ketonuria as a measure of acute starvation/dehydration, and at least a $5 \%(>3 \mathrm{~kg})$ weight loss from the prepregnancy weight. ${ }^{34}$ We will differentiate studies on NVP of pregnancy, and studies on severe form, HG.

\section{Data source and search strategy}

The search will be commenced on 10 February 2019. Initial search throughout the databases will be conducted to collect all the relevant Medical Subject Headings, keywords and free-text words contained in the title and abstract of similar studies. Afterward, we will search for articles in PubMed, CINAHL, EMBASE, EBSCO, Ovid maternity and infant care databases, Cochrane Database of Systematic Reviews, Web of Science and SCOPUS databases using the following search terms: 'Hyperemesis gravidarum', 'HG', 'Severe Nausea and Vomiting' and 'pregnancy outcome'. We will also include the names of LMICs in the search string. The search string was constructed in consultation with medical information specialist and Cochrane Pregnancy and Childbirth Group web page (https://pregnancy.cochrane.org/). The Peer Review of Electronic Search Strategies 2015 guideline statement will be well followed in the process of developing the search string. ${ }^{35}$ The search strategy has been designed and presented with the protocol (see online supplementary file 2). Moreover, cross-references of included articles will be hand searched. Search for grey literature will be carried out by using Google Scholar.

\section{Eligibility criteria}

\section{Inclusion criteria}

- Observational (cross-sectional, case-control, cohort, survey and surveillance report) studies conducted from January 2000 to September 2018 in LMIC reporting the prevalence or burden of hyperemesis or risk factors, or providing enough data to compute these estimates will be included.

- Published articles and grey literature providing statistical data regarding the risk factor associated with hyperemesis incidence in LMIC.

- Studies addressing risk factors and outcome assessments comparing both within women with hyperemesis, and between hyperemesis and non-hyperemesis women will be reviewed.

- Studies published in the English language.

Exclusion criteria

- Studies reported the level of NVP without addressing hyperemesis. 
- Case reports, case series, expert opinion and qualitative articles.

- Full paper that is not accessible even after a request from the authors.

\section{Selection of studies}

Screening and selection process will be done using Covidence web-based software. The title and abstract screening will be done by two (MTD and NBY) independent reviewers. Any disagreement between reviewers will be resolved through consensus and assistance of a third reviewer. Afterward, the full text of eligible articles will be imported to Covidence to determine articles potential for quality assessment and final analysis. Reference lists of selected articles will be checked in order to identify other potential studies of interest.

\section{Quality assessment and data extraction}

The Grading of Recommendations Assessment, Development and Evaluation approach will be followed to rate the quality of scientific evidence in terms of risk of bias, consistency, directness of evidence, the precision of effect of an estimate and publication bias. The quality of evidence for each outcome will fall into one of the four categories from high to very low. In this approach, randomised controlled trials without important limitations constitute highquality evidence. Observational studies without special strengths or important limitations constitute low-quality evidence. However, limitations or special strengths can modify the quality of the evidence. The system offers either strong or weak grades of recommendations. Strong recommendations suggest that the desirable effects of an intervention clearly outweigh the undesirable effects. On the other hand, weak recommendations imply that there is low-quality evidence or the evidence suggests that desirable and undesirable effects are closely balanced. ${ }^{36}$ Two independent reviewers (MTD and NBY) will use the Cochrane collaboration data extraction tool to extract relevant information including the study population, sample size, outcomes, least adjusted determinants of hyperemesis and source of funding. For articles with incomplete data, the corresponding author(s) will be contacted for additional information. Disagreements between the reviewers will be resolved through discussion or involvement of a third reviewer.

\section{Risk of bias}

The Risk of Bias Assessment Tool for Non-randomized Studies will be used to examine the risk of bias. ${ }^{37}$ There are seven domains (ie, confounding, selection of participants, classification of interventions, deviation from intended interventions, missing data, measurement of outcomes, selection of reported result) of bias in the model. Each domain has specific signalling questions, with response options: 'yes', 'probably yes', 'no', 'probably no' or 'no information'. Then the overall judgement on the risk of bias for the outcome and result will be rated as: 'low risk', 'moderate risk', 'serious risk' and 'critical risk' of bias. Importantly, 'low risk' is comparable to the risk of bias in a high-quality randomised trial. The Joanna Briggs Institute critical appraisal checklist will be applied to examine the risk of bias in quasi-randomised controlled trials. ${ }^{38}$

\section{Data synthesis and analysis}

Weighted inverse-variance meta-analysis using fixed-effects and random-effects model will be performed to determine the pooled estimate of HG. If the burden of hyperemesis is measured in different ways among studies, it becomes difficult to run meta-analysis. However, for studies with the same endpoint measurements, we will report the overall mean prevalence of hyperemesis with SD calculated either from SEs or 95\% CI. We will pool the OR and $95 \% \mathrm{CI}$ of studies with identical outcomes. For risk factors, we will show the pooled ORs with 95\% CI. We will use random-effect meta-analysis if considerable heterogeneity of setting, study designs and participants occur.

Effect sizes will be expressed in terms of prevalence, ORs (for categorical data) and weighted mean differences (for continuous data) along with their respective 95\% CIs. To adjust the effect of studies with high or low effect size, a leave-one-out method will be employed. ${ }^{39} 40$ If the normality assumption is fulfilled, arcsine transformation will be carried out. ${ }^{41}$ STATA V.14 will be used to analyse the data.

Heterogeneity among studies will be checked using $\mathrm{T}^{2}$ to determine dispersion. ${ }^{42}$ If substantial heterogeneity exists between studies, random-effect model results will be reported. ${ }^{43}$ Meta-regression analysis will be performed to investigate methodological (ie, study characteristics) and clinical (ie, population characteristics) sources of heterogeneity. Moreover, heterogeneity will be examined manually based on study populations, study area (country), study design and methods to pool estimates.

Funnel plot and Egger's regression statistical test will be applied to check for publication and small sample size bias. Duval and Tweedie trim-and-fill method will be used if publication bias is detected.$^{44}$ Moreover, controlling for potential confounders (demographics, study methods and setting), meta-regression analysis will be conducted.

The findings will be narrated using tables and figures if statistical pooling is not possible due to substantial heterogeneity. The Guidance on the Conduct of Narrative Synthesis in Systematic Reviews will be adapted to develop the synthesis. ${ }^{45}$ First, studies will be grouped and clustered based on their study design, setting (institution based and community based) and the nature of the results being reported. Then the characteristics of the included set of studies, that is, study details and participants will be clustered together and reported in summary tables. The data for prevalence of hyperemesis will be presented separately according to the regions, based on LMIC classified by the world bank. 


\section{Sensitivity analysis}

We will perform a primary analysis of the extracted data. Considering the quality or sample size, studies either can be added or removed to determine whether the changes have any effect on the combined outcome estimate. Studies noticed to be of lower quality will be removed and the analysis will be run again. If the analysis is robust, then there will be little changes in the overall outcome estimate. Moreover, performing random and fixed-effects model, the model that best fits will be used.

\section{Subgroup analysis}

If sufficient data are available, subgroup analysis will be conducted to explore the variation with predetermined factors (eg, geographical distribution of the LMIC, parity, number of fetuses, socioeconomic profile of the country).

\section{Public and patient involvement statement}

The study will not include patients as study participants. We will use published articles to synthesise new evidence on HG.

\section{Ethics and dissemination}

The result will be published in a peer-reviewed scientific journal and will be presented at scientific conferences and public press.

\section{DISCUSSION}

This protocol is rigorously developed and designed specifically to assess the prevalence, risk factors and outcomes of HG in LMIC. Given the scarcity of evidence, it would be helpful for researchers, policy-makers, government and non-governmental organisations for improving maternal and child healthcare in LMIC.

\section{Potential methodological amendments}

If protocol modifications are required, the authors will include the detailed description of any changes along with a justification during the publication of the review.

\section{Author affiliations \\ ${ }^{1}$ Department of Midwifery, Debre Berhan University, Debre Berhan, Ethiopia ${ }^{2}$ Department of Nursing and Midwifery, Wollega University, Nekemte, Ethiopia ${ }^{3}$ Department of Midwifery, College of Medicine and Health science, Woldia University, Woldia, Ethiopia \\ ${ }^{4}$ Department of Midwifery, Debre Markos University, Debre Markos, Ethiopia ${ }^{5}$ Department of Nursing, Debre Berhan University, Debre Berhan, Ethiopia ${ }^{6}$ Department of Epidemiology, University of Groningen, University Medical Center Groningen, Groningen, The Netherlands}

Contributors MTD conceived and designed the study. MTD developed the search strategy. MAM, TT, NBY, MD, TD and MTD wrote and prepared the protocol. MTD, NBY and TD planned the data extraction and aimed to perform the analysis. TT and TD provided critical comments. All authors read and critically revised the protocol.

Funding The authors have not declared a specific grant for this research from any funding agency in the public, commercial or not-for-profit sectors.

Competing interests None declared.

Patient consent for publication Not required.

Ethics approval Formal ethical approval is not required as primary data collection will not be performed.
Provenance and peer review Not commissioned; externally peer reviewed.

Open access This is an open access article distributed in accordance with the Creative Commons Attribution Non Commercial (CC BY-NC 4.0) license, which permits others to distribute, remix, adapt, build upon this work non-commercially, and license their derivative works on different terms, provided the original work is properly cited, appropriate credit is given, any changes made indicated, and the use is non-commercial. See: http://creativecommons.org/licenses/by-nc/4.0/.

\section{REFERENCES}

1. O'Brien B, Evans M, White-McDonald E. Isolation from "being alive": coping with severe nausea and vomiting of pregnancy. Nurs Res 2002;51:302-8.

2. Gadsby R, Barnie-Adshead T. Severe nausea and vomiting of pregnancy: should it be treated with appropriate pharmacotherapy? Obstet Gynecol 2011;13:107-11.

3. Gazmararian JA, Petersen R, Jamieson DJ, et al. Hospitalizations during pregnancy among managed care enrollees. Obstet Gynecol 2002;100:94-100.

4. Lee NM, Saha S. Nausea and vomiting of pregnancy. Gastroenterol Clin North Am 2011;40:309-34.

5. MacGibbon K, Fejzo M, Mullin P. Mortality secondary to hyperemesis gravidarum: a case report. 2015;7:2.

6. Fell DB, Dodds L, Joseph KS, et al. Risk factors for hyperemesis gravidarum requiring hospital admission during pregnancy. Obstet Gynecol 2006;107:277-84.

7. Einarson TR, Piwko C, Koren G. Quantifying the global rates of nausea and vomiting of pregnancy: a meta analysis. J Popul Ther Clin Pharmacol 2013;20:e171-83.

8. Kejela G, Getu S, Gebretsdik T, et al. Prevalence of Hyperemesis Gravidarum and Associated Factors in Arba Minch General Hospital, Gamo Gofa Zone, Southern Ethiopia. Clin Mother Child Health 2018;15.

9. Mahmoud G. Prevalence and Risk Factors of Hyperemesis Graviderum Among Egyptian Pregnant Woman at the Woman's Health Center. Med J Cairo Univ 2012;80.

10. London V, Grube S, Sherer DM, et al. Hyperemesis Gravidarum: A Review of Recent Literature. Pharmacology 2017;100(3-4):161-71.

11. Vlachodimitropoulou Koumoutsea E, VlachodimitropoulouKoumoutsea E, Gosh S, Manmatharajah B, et al. Pregnancy outcomes in severe hyperemesis gravidarum in a multi-ethnic population. J Obstet Gynaecol 2013;33:455-8.

12. Bailit JL. Hyperemesis gravidarium: epidemiologic findings from a large cohort. Am J Obstet Gynecol 2005;193:811-4.

13. Fejzo MS, Ingles SA, Wilson M, et al. High prevalence of severe nausea and vomiting of pregnancy and hyperemesis gravidarum among relatives of affected individuals. Eur J Obstet Gynecol Reprod Biol 2008;141:13-17.

14. Boelig RC, Barton SJ, Saccone G, et al. Interventions for treating hyperemesis gravidarum. Cochrane Database Syst Rev 2013.

15. Aksoy AN. Hyperemesis Incidence in Planned versus Unplanned Pregnancy. Eurasian J Med 2008;40:72.

16. Kjeldgaard HK, Eberhard-Gran M, Benth JŠ, et al. History of depression and risk of hyperemesis gravidarum: a population-based cohort study. Arch Womens Ment Health 2017;20:397-404.

17. Fiaschi L, Nelson-Piercy C, Tata LJ. Hospital admission for hyperemesis gravidarum: a nationwide study of occurrence, reoccurrence and risk factors among 8.2 million pregnancies. Hum Reprod 2016;31:1675-84.

18. Enakpene CA, Arya S, Dalloul M, et al. Dysmenorrhea as a risk factor for hyperemesis gravidarum. Fertil Steril 2012;98:S201.

19. Clark S, Hughes B, McDonald SS. The impact of nausea and vomiting of pregnancy on quality of life: report of a national consumer survey and recommendations for improving care. Obstet Gynecol Surv 2013;68:S1-S10.

20. Lacasse A, Rey E, Ferreira E, et al. Nausea and vomiting of pregnancy: what about quality of life? BJOG Int J Obstet Gy 2008;115:1484-93.

21. Selitsky T, Chandra P, Schiavello HJ. Wernicke's encephalopathy with hyperemesis and ketoacidosis. Obstet Gynecol 2006;107(2 Pt 2):486-90.

22. Buchanan GM, Franklin V. Hamman and Boerhaave syndromes - diagnostic dilemmas in a patient presenting with hyperemesis gravidarum: a case report. Scott Med J 2014;59:e12-e16.

23. Heitmann K, Holst L, Lupattelli $A$, et al. Treatment of nausea in pregnancy: a cross-sectional multinational web-based study of 
pregnant women and new mothers. BMC Pregnancy Childbirth 2015;15:321.

24. Dean C, Bannigan K, Marsden J. Reviewing the effect of hyperemesis gravidarum on women's lives and mental health. $\mathrm{Br} \mathrm{J}$ Midwifery 2018;26:109-19.

25. Mullin PM, Bray A, Schoenberg F, et al. Prenatal exposure to hyperemesis gravidarum linked to increased risk of psychological and behavioral disorders in adulthood. J Dev Orig Health Dis 2011;2:200-4.

26. Wood H, McKellar LV, Lightbody M. Nausea and vomiting in pregnancy: blooming or bloomin'awful? A review of the literature. Women Birth 2013;26:100-4.

27. Heitmann K, Nordeng H, Havnen GC, et al. The burden of nausea and vomiting during pregnancy: severe impacts on quality of life, daily life functioning and willingness to become pregnant againresults from a cross-sectional study. BMC Pregnancy Childbirth 2017;17:75.

28. Piwko C, Ungar WJ, Einarson TR, et al. The weekly cost of nausea and vomiting of pregnancy for women calling the Toronto Motherisk Program. Curr Med Res Opin 2007;23:833-40.

29. Piwko C, Koren G, Babashov V, et al. Economic burden of nausea and vomiting of pregnancy in the USA.J Popul Ther Clin Pharmacol 2013;20:e149-e60.

30. Moher D, Liberati A, Tetzlaff J, et al. Reprint-preferred reporting items for systematic reviews and meta-analyses: the PRISMA statement. Phys Ther 2009;89:873-80.

31. Shamseer $L$, Moher D, Clarke M, et al. Preferred reporting items for systematic review and meta-analysis protocols (PRISMA-P) 2015: elaboration and explanation. BMJ 2015;349:g7647.

32. Moher D, Shamseer L, Clarke M, et al. Preferred reporting items for systematic review and meta-analysis protocols (PRISMA-P) 2015 statement. Syst Rev 2015;4:1.

33. The World Bank. New country classifications by income level: 2018. World Bank Blogs, 2018. Available: https://blogs.worldbank.org/ opendata/new-country-classifications-income-level-2018-2019
34. Chia CF, Lai JH, Cheung PK, et al. Dysmenorrhoea among Hong Kong university students: prevalence, impact, and management. Hong Kong Med J 2013;19:222-8.

35. McGowan J, Sampson M, Salzwedel DM, et al. PRESS peer review of electronic search strategies: 2015 guideline statement. J Clin Epidemiol 2016;75:40-6.

36. Dijkers M. Introducing GRADE: a systematic approach to rating evidence in systematic reviews and to guideline development. $K T$ Update 2013;1:1-9.

37. Sterne JA, Hernán MA, Reeves BC, et al. ROBINS-I: a tool for assessing risk of bias in non-randomised studies of interventions. BMJ 2016;355:i4919.

38. The Joanna Briggs Institute (JBI). Checklist for randomized controlled trials. 2017.

39. Tsumoto S, Hirano S. (eds). Formal Analysis of Leave-One-Out Methods Based on Decremental Sampling Scheme. Web Intelligence (WI) and Intelligent Agent Technologies (IAT), 2014 IEEE/WIC/ACM International Joint Conferences on: IEEE, 2014

40. Gonzales DA, Norsworthy KJ, Kern SJ, et al. A meta-analysis of $\mathrm{N}$-acetylcysteine in contrast-induced nephrotoxicity: unsupervised clustering to resolve heterogeneity. BMC Med 2007;5:32.

41. M-I L, H-z T, Zhou Q, et al. Realizing the meta-analysis of single rate in R software. J Evid Based Med 2013;13:181-4.

42. Borenstein M, Higgins JP, Hedges LV, et al. Basics of meta-analysis: $\mathrm{I}^{2}$ is not an absolute measure of heterogeneity. Res Synth Methods 2017:8:5-18.

43. Bown MJ, Sutton AJ. Quality control in systematic reviews and metaanalyses. Eur J Vasc Endovasc Surg 2010;40:669-77.

44. Duval S, Tweedie R. Trim and fill: a simple funnel-plot-based method of testing and adjusting for publication bias in meta-analysis. Biometrics 2000;56:455-63.

45. Popay J, Roberts H, Sowden A, et al. Guidance on the conduct of narrative synthesis in systematic reviews. A product from the ESRC methods programme Version 2006;1:b92. ESRC. 